\title{
The Chinese Remainder Theorem for strongly semisimple MV-algebras and lattice-groups.
}

For Tonino

\author{
Vincenzo Marra \\ Dipartimento di Matematica "Federigo Enriques" \\ Università degli Studi di Milano \\ Via Cesare Saldini 50, 20133 Milano, Italy
}

\begin{abstract}
An MV-algebra (equivalently, a lattice-ordered Abelian group with a distinguished order unit) is strongly semisimple if all of its quotients modulo finitely generated congruences are semisimple. All MV-algebras satisfy a Chinese Reminder Theorem, as was first shown by Keimel four decades ago in the context of latticegroups. In this note we prove that the Chinese Remainder Theorem admits a considerable strengthening for strongly semisimple structures.
\end{abstract}

Key words: Lattice-ordered Abelian group, MV-algebra, strong order unit, semisimple algebra, spectral space, Zariski topology, hull-kernel topology, Chinese Remainder Theorem.

2010 MSC: Primary: 06F20. Secondary: 06D35.

\section{Introduction.}

We assume familiarity with MV-algebras [3] and lattice-ordered Abelian groups [2], which we write in additive notation and call $\ell$-groups for short. The mathematics in this note can be presented either in the language of $\mathrm{MV}$-algebras, or in the categorically equivalent language of $\ell$-groups with a strong order unit, thanks to [19, Theorem 3.9]. We choose $\ell$-groups because we are going to use [2, Chapitre 10] as a convenient source for Keimel's spectral spaces of lattice-groups. We write ' $\ell$-ideal' to mean 'order-convex sublattice subgroup'; such are precisely the kernels of lattice-group homomorphisms, which are in bijection with congruences. If $I$ is an $\ell$-ideal of $G$ and $g \in G$, we write $G / J$ for the quotient $\ell$-group, and $[g]_{I} \in G / I$ for the congruence class of $g$ modulo $I$, i.e. for the coset $g / I:=g+I:=\{g+h \mid h \in I\}$. It is clear that an arbitrary intersection of $\ell$-ideals is again an $\ell$-ideal. The $\ell$-ideal generated by a subset $S \subseteq G$ of an $\ell$-group $G$ is therefore defined as the intersection of all $\ell$-ideals of $G$ containing $S$.

In his work on sheaf-theoretic representation of $\ell$-groups [17], [2, Chapitre 10], Keimel used the following

Chinese Remainder Theorem 1.1. Let $\left\{I_{i}\right\}_{i=1}^{n}$ be a finite collection of $\ell$-ideals of an $\ell$-group $G$, and suppose that $\left\{g_{i}\right\}_{i=1}^{n}$ is a finite subset of $G$ such that

$$
\left[g_{i}\right]_{I_{i} \vee I_{j}}=\left[g_{j}\right]_{I_{i} \vee I_{j}}
$$

for each $i, j \in\{1, \ldots, n\}$, where $I_{i} \vee I_{j}$ denotes the $\ell$-ideal of $G$ generated by $I_{i} \cup I_{j}$. Then there exists an element $g \in G$ such that $[g]_{I_{i}}=\left[g_{i}\right]_{I_{i}}$ for each $i \in\{1, \ldots, n\}$.

Proof. This is $[2,10.6 .3]$.

Email addresses: vincenzo.marra@unimi.it (Vincenzo Marra)

Preprint submitted to Mathematica Slovaca, Antonio Di Nola's Festschrift, on May 25, 2013 
In one form or another, this result plays a rôle in any sheaf-theoretic representation of MV-algebras and lattice-groups. In Yang's Ph.D. Thesis [23, Proposition 5.1.2, see also Remark 5.3.12], Keimel's Chinese Remainder Theorem is used. Dubuc's and Poveda's Pullback-Pushout Lemma [7, 3.11] is a consequence of the Chinese Remainder Theorem. In Filipoiu's and Georgescu's paper [10] the result, while not featuring explicitly, floats beneath the surface - see e.g. [10, Proposition 2.16]. Schwartz [21, Theorem 3.1] invokes Keimel's result to obtain his representation; on the other hand, Rump and Yang [20] use Keimel's sheaftheoretic representation without employing the result directly. In [9, Theorem 2.6], Ferraioli and Lettieri prove the Chinese Remainder Theorem for MV-algebras. They do not use nor mention Keimel's result; $c f$. also [6]. Let us also point out that sheaf-theoretic representations and Chinese Remainder Theorems were studied at the level of general algebra by Vaggione in [22], whose results extend the previous ones by Krauss and Clark [18]; see also Cornish's earlier paper [5] in the same direction. For a unified approach to sheaf-theoretic representations of MV-algebras using Stone-Priestley duality for distributive lattices, see [11].

Here we prove that strongly semisimple unital $\ell$-groups and MV-algebras enjoy a considerably stronger version of the general Chinese Remainder Theorem. Let us recall some basic facts first.

An $\ell$-ideal $\mathfrak{p}$ of an $\ell$-group $G$ is prime $[2,2.4 .1]$ if it is proper $(i . e . \neq G$ ), and the quotient $G / \mathfrak{p}$ is totally ordered; and it is maximal if it is proper, and maximal with respect to inclusion. It is elementary that each maximal $\ell$-ideal is prime. Principal (=singly generated) and finitely generated $\ell$-ideals can be shown to coincide.

A unital $\ell$-group $(G, u)$ is an $\ell$-group equipped with a (strong order) unit [2, 2.2.12]: a non-negative element $u \in G$ whose positive multiples eventually dominate each element of $G$. The unital setting is convenient because it ensures that spaces of prime congruences are compact, see Section 2. By a unital $\ell$-subgroup of the unital $\ell$-group $(G, u)$ we mean an $\ell$-subgroup of $G$ that contains $u$. By ' $\ell$-homomorphism' we mean 'latticegroup homomorphism', and by 'unital $\ell$-homomorphism' we mean 'unit-preserving $\ell$-homomorphism'

The Archimedean property plays a substantive rôle in this paper. The unital $\ell$-group $(G, u)$ is simple if it has no proper, non-trivial $(i . e . \neq\{0\}) \ell$-ideals. It is semisimple if the intersection of all its maximal $\ell$-ideals is the trivial $\ell$-ideal $\{0\}$; equivalently, if it is a unital subdirect product of simple unital $\ell$-groups; equivalently, if it is Archimedean: for all $g, h \in G$ with $0<g \leqslant h$, there is an integer $m \geqslant 0$ such that $m g \$ h$. Not all of these formulations of the Archimedean property are equivalent in the absence of a unit; see Lemma 2.4 and Remark 2.5 below. Finally, $(G, u)$ is strongly semisimple if each one of its quotients modulo a principal $\ell$-ideal is semisimple. Since we are not excluding the trivial principal $\ell$-ideal $\{0\}$ in the preceding definition, if $G$ is strongly semisimple then it is semisimple.

Remark 1.2. In the literature on MV-algebras, the term "strongly semisimple" first appeared in [7]. However, the closely related concept of "logically complete MV-algebras" was already introduced by Belluce and Di Nola in [1]. By contrast, to the best of our knowledge strong semisimplicity has not been considered in the literature on lattice-groups.

Now let us consider a unital $\ell$-group $(G, u)$ with unit $u$, and let Max $G$ denote its set of maximal $\ell$-ideals. The subsets of the form $\{\mathfrak{m} \in \operatorname{Max} G \mid \mathfrak{m} \supseteq I\}$, as $I$ ranges over all possible $\ell$-ideals of $G$, are the closed sets of a compact Hausdorff topology on Max $G$, known as the hull-kernel topology, cf. [12, p. 111]. A construction of Yosida [24], significantly generalised in [13], associates to each element $g \in G$ a continuous function $\widehat{g}: \operatorname{Max} G \rightarrow \mathbb{R}$. In the unital case, the crux of the matter is the classical result by Hölder [15] that each quotient $\left(G / \mathfrak{m},[u]_{\mathfrak{m}}\right)$, with $\mathfrak{m}$ a maximal $\ell$-ideal of $G$, admits a unique unit-preserving injective $\ell$-homomorphism $\mathfrak{h}_{\mathfrak{m}}:\left(G / \mathfrak{m},[u]_{\mathfrak{m}}\right) \hookrightarrow(\mathbb{R}, 1)$. Then $\widehat{g}(\mathfrak{m})$ is the unique real number $\mathfrak{h}_{\mathfrak{m}}\left([g]_{\mathfrak{m}}\right)$. See Lemma 2.3 below. A subset $Z \subseteq \operatorname{Max} G$ is a principal zero set if it is of the form $Z=\widehat{g}^{-1}(0)$ for some $g \in G$; equivalently, $Z=\{\mathfrak{m} \in \operatorname{Max} G \mid g \in \mathfrak{m}\}$. It is an exercise to check that the principal zero sets form a basis of closed sets for the hull-kernel topology of $\operatorname{Max} G$. Main result:

Theorem. Let $(G, u)$ be a unital $\ell$-group, and let $\left\{Z_{i}\right\}_{i=1}^{n}$ be a finite collection of principal zero sets of Max $G$. Suppose that $\left\{g_{i}\right\}_{i=1}^{n} \subseteq G$ is compatible with $\left\{Z_{i}\right\}_{i=1}^{n}$, meaning that $\widehat{g}_{i}(\mathfrak{m})=\widehat{g}_{j}(\mathfrak{m})$ for each $i, j \in\{1, \ldots, n\}$ and each $\mathfrak{m} \in Z_{i} \cap Z_{j}$. If $(G, u)$ is strongly semisimple, there exists an element $g \in G$ such that $\widehat{g}(\mathfrak{m})=\widehat{g}_{i}(\mathfrak{m})$ for each $i \in\{1, \ldots, n\}$ and each $\mathfrak{m} \in Z_{i}$. If, moreover, $\bigcup_{i=1}^{n} Z_{i}=\operatorname{Max} G$, such an element $g$ is unique. 
We prove the theorem in Section 3, after some preliminaries in Section 2. We will see in due course that the theorem above is a Chinese Remainder Theorem for semisimple structures, stated in the functional language of the Yosida representation. Indeed, while the result is formulated here in terms of Max $G$, we prove as Lemma 3.5 an equivalent statement for the spectral space of prime $\ell$-ideals of $G$. Lemma 3.5 is a (new) strengthening for strongly semisimple structures of the (known) Patching Lemma 2.2 that holds in full generality. The Patching Lemma, in turn, is the dual spectral version of Keimel's Chinese Remainder Theorem 1.1 for lattice-groups. Symmetrically, the dual algebraic version of Lemma 3.5, given below as Theorem 3.6, provides a (new) Chinese Remainder Theorem for strongly semisimple structures. Our reformulation of the theorem above in the spectral language of Lemma 3.5 requires the characterisation of strongly semisimple structures in terms of the topology of the prime spectrum. This we achieve in Corollary 3.4; a similar result for Archimedean $\ell$-groups has been previously obtained by Yang [23, Proposition 5.3.1].

\section{Preliminary results.}

Throughout this note we let $(G, u)$ denote a unital $\ell$-group.

\subsection{Spectral spaces of unital lattice-ordered Abelian groups.}

Consider the family Idl $G$ of all $\ell$-ideals of $G$. Then, as proved in [2, 2.2.7 and 2.2.9], Idl $C$ is a complete bounded distributive lattice under the operations $I \wedge J:=I \cap J$ and $I \vee J:=\langle I \cup J\rangle$, where $I, J \in \operatorname{Idl} G$ and $\langle S\rangle$ denotes the intersection of all $\ell$-ideals of $G$ containing $S$. Let Prin $G$ denote the subset of Idl $G$ consisting of principal $\ell$-ideals. Then $\operatorname{Prin} G$ is in fact a sublattice of $\operatorname{Idl} G,[2,2.2 .11]$. Write Spec $G$ for the set of prime $\ell$-ideals of $G$, and $\operatorname{Max} G \subseteq \operatorname{Spec} G$ for the set of all maximal $\ell$-ideals of $G$.

For any $R \subseteq G$, set

$$
\mathbb{V}(R):=\{\mathfrak{p} \in \operatorname{Spec} G \mid \mathfrak{p} \supseteq R\} .
$$

Symmetrically, for any $S \subseteq \operatorname{Spec} G$, set

$$
\mathbb{I}(S):=\{g \in G \mid g \in \mathfrak{p} \text { for each } \mathfrak{p} \in S\}=\bigcap S .
$$

For any set $X$, we let $2^{X}$ denote the set of the subsets of $X$.

Lemma 2.1 (Keimel's Spectral Topology). Consider the functions $\mathbb{V}: 2^{G} \longrightarrow 2^{\operatorname{Spec} G}$ and $\mathbb{I}: 2^{\operatorname{Spec} G} \longrightarrow 2^{G}$ given by $(1-2)$.

1. The pair $(\mathbb{V}, \mathbb{I})$ yields a (contravariant) Galois connection between the $2^{G}$ and $2^{\mathrm{Spec} G}$. That is,

$$
R \subseteq \mathbb{I}(S) \text { if, and only if, } S \subseteq \mathbb{V}(R)
$$

for all $R \subseteq G$ and $S \subseteq \operatorname{Spec} G$.

2. $\mathbb{V}$ reverses arbitrary unions to intersections: $\mathbb{V}\left(\bigcup_{i \in D} R_{i}\right)=\bigcap_{i \in D} \mathbb{V}\left(R_{i}\right)$, where $R_{i}$ is a subset of $G$, and $D$ is an arbitrary index set. Similarly, $\mathbb{I}$ reverses arbitrary unions to intersections: $\mathbb{I}\left(\bigcup_{i \in D} S_{i}\right)=$ $\bigcap_{i \in D} \mathbb{I}\left(S_{i}\right)$, where $S_{i}$ is a subset of $\operatorname{Spec} G$, and $D$ is an arbitrary index set.

3. The fixed subsets of $G$, i.e. the ones for which $\mathbb{I}(\mathbb{V}(R))=R$, are precisely its $\ell$-ideals.

4. The fixed subsets of $\operatorname{Spec} G$, i.e. the ones for which $\mathbb{V}(\mathbb{I}(S))=S$, are precisely the vanishing loci of $\ell$-ideals, that is, those of the form

$$
\mathbb{V}(I), \text { for some } I \in \operatorname{Idl} G \text {. }
$$

5. The sets of the form $(*)$ are the closed sets of a topology ${ }^{1}$ on $\operatorname{Spec} G$, whose associated closure operator is given by $\mathrm{cl}:=\mathbb{V} \circ \mathbb{I}: 2^{\operatorname{Spec} G} \longrightarrow 2^{\operatorname{Spec} G}$.

\footnotetext{
${ }^{1}$ This topology on $\operatorname{Spec} G$ is variously called its Zariski, or spectral, or hull-kernel topology.
} 
6. The space $\operatorname{Spec} G$ is spectral: ${ }^{2}$ it is $T_{0}$, compact, its compact open subsets form a basis of opens sets stable under finite intersections, and it is sober - i.e. every non-empty, closed subset that cannot be written as the union of two proper closed subsets, has a dense point.

7. The compact and open subsets of $\operatorname{Spec} G$ are precisely the complements of those of the form $\mathbb{V}(P)$, for $P$ a principal $\ell$-ideal of $G$. Hence, the lattice Prin $G$ is isomorphic and anti-isomorphic to the lattices of compact open and co-compact ${ }^{3}$ closed subsets of $\operatorname{Spec} G$, respectively. ${ }^{4}$

8. Equip $\operatorname{Max} G$ with the subspace topology that it inherits from $\operatorname{Spec} G$. Then $\operatorname{Max} G$ is a compact Hausdorff space.

Proof. Item 1 is an exercise. Item 2 is a general property of Galois connections, see e.g. [8] for background. Items 3 and 4 are easy to prove using the fact that each $\ell$-ideal is an intersection of the prime $\ell$-ideals that contain it $[2,2.5 .5]$. Items $5-7$ are proved in $[2,10.1 .2-10.1 .10]$. Item 8 is $[2,10.2 .5]$.

It is immediate to describe the closure operator cl: $2^{\operatorname{Spec} G} \longrightarrow 2^{\operatorname{Spec} G}$ in terms of intersections of primes. For any subset $S \subseteq \operatorname{Spec} G$, we have

$$
\operatorname{cl} S=\{\mathfrak{p} \in \operatorname{Spec} G \mid \mathfrak{p} \supseteq \bigcap S\}
$$

Indeed, by definition $\operatorname{cl} S=\mathbb{V}(\mathbb{I}(S))=\{\mathfrak{p} \in \operatorname{Spec} G \mid \mathfrak{p} \supseteq \mathbb{I}(S)\}$, and $\mathbb{I}(S)=\bigcap S$.

\subsection{The Patching Lemma.}

The Patching Lemma 2.2. Let $\left\{U_{i}\right\}_{i=1}^{n}$ be a finite collection of closed subsets of Spec $G$, and suppose that $\left\{g_{i}\right\}_{i=1}^{n}$ is a finite subset of $G$ such that $\left[g_{i}\right]_{\mathfrak{p}}=\left[g_{j}\right]_{\mathfrak{p}}$ for each $i, j \in\{1, \ldots, n\}$ and each $\mathfrak{p} \in U_{i} \cap U_{j}$. Then there exists an element $g \in G$, such that $[g]_{\mathfrak{p}}=\left[g_{i}\right]_{\mathfrak{p}}$ for each $i \in\{1, \ldots, n\}$ and each $\mathfrak{p} \in U_{i}$.

Proof. This is a direct dualisation of the Chinese Remainder Theorem 1.1 to Spec G, using Lemma 2.1.

\subsection{The Archimedean property, semisimplicity, and the maximal spectrum.}

For $X$ a topological space, a subset $S \subseteq \mathrm{C}(X)$ is said to separate the points (of $X$ ) if for each $x \neq y \in X$ there is $f \in S$ with $f(x)=0$ and $f(y) \neq 0$.

Lemma 2.3 (Hölder's Theorem [15], and Yosida's Theorem $\left.{ }^{5}[24]\right)$. Let $(G, u)$ be a unital $\ell$-group.

1. If $G$ is Archimedean and totally ordered, then there is a unique unital injective $\ell$-homomorphism $(G, u) \hookrightarrow(\mathbb{R}, 1)$.

2. If $\mathfrak{m} \in \operatorname{Max} G$, then there is a unique unital injective $\ell$-homomorphism $\mathfrak{h}_{\mathfrak{m}}:(G / \mathfrak{m}, u / \mathfrak{m}) \hookrightarrow(\mathbb{R}, 1)$.

3. If $g \in G$, the function

$$
\begin{aligned}
\widehat{g} & : \operatorname{Max} G \longrightarrow \mathbb{R} \\
\mathfrak{m} & \in \operatorname{Max} G \longmapsto \mathfrak{h}_{\mathfrak{m}}(g / \mathfrak{m}) \in \mathbb{R},
\end{aligned}
$$

where $\mathfrak{h}_{\mathfrak{m}}$ is given by 2 , is continuous with respect to the Euclidean topology of $\mathbb{R}$.

\footnotetext{
${ }^{2}$ In the sense of Hochster [14, p. 43].

${ }^{3}$ Throughout, we write 'co-compact set' to mean 'complement of a compact set'.

${ }^{4}$ In particular, the space prime lattice ideals of the bounded distributive lattice Prin $G$, endowed with the usual Stone topology — see e.g. [16] — is homeomorphic to $\operatorname{Spec} G$.

${ }^{5}$ While [24, Theorems 1 and 2] prove more than our (iii-iv), that is all we need in this paper.
} 
4. Let $X$ be a compact Hausdorff space, and assume that $G \subseteq \mathrm{C}(X)$ is an $\ell$-subgroup, and that $u=1_{X}$. If $G$ separates the points of $X$, then $\operatorname{Max} G$ is homeomorphic to $X$ via the map

$$
\mathfrak{m} \in \operatorname{Max} G \longmapsto x(\mathfrak{m}) \in X,
$$

where $x(\mathfrak{m})$ is the unique point of $X$ in the set $\bigcap\left\{f^{-1}(0) \mid f \in \mathfrak{m}\right\}$.

Proof. 1 . This is $[2,2.6 .3]$, with the additional observation that the unital assumption makes the embedding unique.

2. Observe that $\left(G / \mathfrak{m},[u]_{\mathfrak{m}}\right)$ is simple, because $\mathfrak{m}$ is maximal, and the $\ell$-ideals of $G / \mathfrak{m}$ are in one-one inclusion-preserving correspondence with the $\ell$-ideals of $G$ containing $\mathfrak{m}[2,2.3 .8]$. Simplicity entails easily that $G / \mathfrak{m}$ is Archimedean and totally ordered, so 1 applies.

3. This was first proved in [24], for unital lattice-ordered vector spaces (known as vector lattices). The proof for $\ell$-groups is a straightforward adaptation of Yosida's argument in [24], using Hölder's Theorem (1-2) in place of his [24, Lemma 2].

4. Essentially [24, Theorem 4].

Lemma 2.4. For any unital $\ell$-group $(G, u)$, the following are equivalent.

1. $(G, u)$ is semisimple.

2. $G$ is Archimedean.

3. The radical of $G$ is trivial, that is, $\operatorname{Rad} G:=\bigcap \operatorname{Max} G=\{0\}$.

Proof. The equivalence of 1 and 2 is an immediate consequence of the fact that a quotient $G / I$, for $I$ a proper $\ell$-ideal of $G$, is simple if, and only if, $I$ is maximal. And this, in turn, follows at once from [2, 2.3.8]. The equivalence of 2 and 3 was first proved, for unital vector lattices, in [25, Theorem 1]. The proof for unital $\ell$-groups is a straightforward adaptation using Hölder's Theorem (Lemma 2.3.(1-2)) in place of [25, Lemma 1]. The present lemma is also proved for MV-algebras in [3, 3.6.1 and 3.6.4].

Remark 2.5. Caution: the equivalence of 2 and 3 in Lemma 2.4 fails in the absence of a unit. See [25, §3] for an early example due to Nakayama.

\section{Proof of theorem.}

We begin by adding to Lemma 2.4 a spectral characterisation of the Archimedean property.

Remark 3.1. The following lemma should be compared to Yang's aforementioned result [23, Proposition 5.3.1].

Lemma 3.2. For any unital $\ell$-group $(G, u)$, the following are equivalent.

1. $(G, u)$ is semisimple.

2. $\operatorname{Max} G$ is dense in $\operatorname{Spec} G$.

Proof. First suppose that $G$ is not semisimple, hence not Archimedean by Lemma 2.4. There exist $g, h \in G$ with $0<n g \leqslant h$ for all integers $n \geqslant 1$. Then $\mathbb{V}(g) \supseteq \operatorname{Max} G$. Indeed, assume by way of contradiction that $[g]_{\mathfrak{m}}>0$ for some $\mathfrak{m} \in \operatorname{Max} G$. By Lemma 2.3.2, $[g]_{\mathfrak{m}},[h]_{\mathfrak{m}} \in \mathbb{R}$ to within the unique unital $\ell$-embedding $\mathfrak{h}_{\mathfrak{m}}$, and $0<[g]_{\mathfrak{m}} \leqslant[h]_{\mathfrak{m}}$. By the Archimedean property of $\mathbb{R}$, there is an integer $n_{0} \geqslant 1$ with $n_{0}[g]_{\mathfrak{m}} \geqslant[h]_{\mathfrak{m}}$, whence $n_{0} g \nless h$ in $G$, contradiction. This proves $\mathbb{V}(g) \supseteq \operatorname{Max} G$. Now, since $\mathbb{V}(g)$ is closed, the closure of $\operatorname{Max} G$ is contained in $\mathbb{V}(g)$. And since $g \neq 0$ and $\bigcap \operatorname{Spec} G=\{0\}[2,10.1 .8]$, there is $\mathfrak{p} \in G$ with $[g]_{\mathfrak{p}} \neq 0$. In other words, $\mathbb{V}(g) \subset \operatorname{Spec} G$. In conclusion, $\operatorname{Spec} G \supset \mathbb{V}(g) \supseteq \operatorname{cl} \operatorname{Max} G$, and $\operatorname{Max} G$ is not dense in Spec $G$.

Conversely, suppose $\operatorname{cl} \operatorname{Max} G \subset \operatorname{Spec} G$. The compact open sets $\left\{\mathbb{V}(g)^{\mathrm{c}} \mid g \in f\right\}$, where $\mathbb{V}(g)^{\mathrm{c}}$ is the complement of $\mathbb{V}(g)$ in $\operatorname{Spec} G$, form a basis of $\operatorname{Spec} G$, by Lemma 2.1. Hence there is $S \subseteq G$ such that the 
open set $O:=(\operatorname{cl} \operatorname{Max} G)^{\mathrm{c}}$ may be written as $O=\bigcup_{g \in S} \mathbb{V}(g)^{\mathrm{c}}$. Therefore, $\mathbb{V}(g) \supseteq \operatorname{Max} G$ for each $g \in S$. Since $O$ is non-empty, we must have $\mathbb{V}\left(g_{0}\right)^{\mathrm{c}} \neq \emptyset$ for some $g_{0} \in S$, so that $g_{0} \neq 0$. But then, since $g_{0} \in \mathfrak{m}$ for each $\mathfrak{m} \in \operatorname{Max} G$, we have $0 \neq g_{0} \in \operatorname{Rad} G=\bigcap \operatorname{Max} G \neq\{0\}$. By Lemma 2.4, $G$ is not semisimple.

Lemma 3.3. Let $(G, u)$ be a unital $\ell$-group, and let I be an $\ell$-ideal of $G$. Then $\operatorname{Spec}(G / I)$ is homeomorphic to $\mathbb{V}(I)$, equipped with the subspace topology it inherits from $\operatorname{Spec} G$.

Proof. By $[2,2.3 .8]$, there is a one-one inclusion preserving correspondence between the $\ell$-ideals of $G$ containing $I$, and the $\ell$-ideals of $G / I$. Specifically, the correspondence is given by

$$
J \supseteq I \stackrel{\rho}{\longmapsto} J / I:=\left\{[j]_{I} \mid j \in J\right\} .
$$

The bijection $\rho$, being inclusion-preserving, restricts to a bijection between maximal $\ell$-ideals of $G$ containing $I$, and maximal $\ell$-ideals of $G / I$. To see that $\rho$ also restricts to a bijection between prime $\ell$-ideals, we use the result in universal algebra $[4,3.11]$ that $G / J \cong(G / I) /(J / I) \cong(G / I) / \rho(J)$. Since $G / J$ is totally ordered if, and only if, $J$ is prime, $\rho(J)$ is prime if, and only if, $J$ is prime. Since $\mathbb{V}(I)$ consists of the prime $\ell$-ideals of $G$ extending $I$, it is clear by the same token that the subspace topology on $\mathbb{V}(I)$ agrees with the topology on $\operatorname{Spec} G / I$.

Corollary 3.4 (Spectral characterisation of strong semisimplicity). Let $(G, u)$ be a unital $\ell$-group. Then $(G, u)$ is strongly semisimple if, and only if, each closed, co-compact subset $K \subseteq \operatorname{Spec} G$ satisfies $K=$ $\operatorname{cl}(K \cap \operatorname{Max} G)$.

Proof. Combine Lemmata 3.2 and 3.3.

The strongly semisimple Patching Lemma 3.5. Let $(G, u)$ be a unital $\ell$-group, and let $\left\{U_{i}\right\}_{i=1}^{n}$ be a finite collection of closed, co-compact subsets of $\operatorname{Spec} G$. Suppose that $\left\{g_{i}\right\}_{i=1}^{n} \subseteq G$ is such that $\left[g_{i}\right]_{\mathfrak{m}}=\left[g_{j}\right]_{\mathfrak{m}}$ for each $i, j \in\{1, \ldots, n\}$ and each $\mathfrak{m} \in U_{i} \cap U_{j} \cap \operatorname{Max} G$. If $(G, u)$ is strongly semisimple, there exists an element $g \in G$ such that $[g]_{\mathfrak{p}}=\left[g_{i}\right]_{\mathfrak{p}}$ for each $i \in\{1, \ldots, n\}$ and each $\mathfrak{p} \in U_{i}$.

Proof. We set

$$
M_{i}:=U_{i} \cap \operatorname{Max} G \text { for each } i \in\{1, \ldots, n\} .
$$

Let $\mathfrak{p} \in U_{i} \cap U_{j}$ for some $i, j \in\{1, \ldots, n\}$. If we show $\left[g_{i}\right]_{\mathfrak{p}}=\left[g_{j}\right]_{\mathfrak{p}}$, then the Patching Lemma 2.2 applies, and the present lemma follows. Equivalently, upon setting $h:=g_{i}-g_{j}$, we need to show $[h]_{\mathfrak{p}} \neq 0$.

Since closed, co-compact sets are stable under finite intersections (Lemma 2.1.7), $K:=U_{i} \cap U_{j}$ is closed and co-compact. Since $(G, u)$ is strongly semisimple, by Corollary 3.4 we have

$$
K=\operatorname{cl}(K \cap \operatorname{Max} G) .
$$

If we had $[h]_{\mathfrak{m}}=0$ for each $\mathfrak{m} \in K \cap \operatorname{Max} G$, we would infer $[h]_{\mathfrak{p}}=0$, too. Indeed, since $\mathfrak{p} \in \operatorname{cl}(K \cap \operatorname{Max} G)$ by (4), we have $\mathfrak{p} \supseteq \bigcap(K \cap \operatorname{Max} G)$ by (3). Also, since $h \in \mathfrak{m}$ for each $\mathfrak{m} \in K \cap \operatorname{Max} G$, we have $h \in \bigcap(K \cap \operatorname{Max} G)$. Hence $h \in \mathfrak{p}$, that is, $[h]_{\mathfrak{p}}=0$, and the proof is complete.

End of proof of theorem. Let $\left\{h_{i}\right\}_{i=1}^{n} \subseteq G$ be elements such that $Z_{i}=\left\{\mathfrak{m} \in \operatorname{Max} G \mid h_{i} \in \mathfrak{m}\right\}, i=1, \ldots, n$. Set

$$
U_{i}:=\mathbb{V}\left(h_{i}\right), i=1, \ldots, n,
$$

so that $U_{i} \cap \operatorname{Max} G=Z_{i}$. By hypothesis, $\widehat{g}_{i}(\mathfrak{m})=\widehat{g}_{j}(\mathfrak{m})$ for each $i, j \in\{1, \ldots, n\}$ and each $\mathfrak{m} \in Z_{i} \cap Z_{j}$, i.e. $\left[g_{i}\right]_{\mathfrak{m}}=\left[g_{j}\right]_{\mathfrak{m}}$ for each $i, j \in\{1, \ldots, n\}$ and each $\mathfrak{m} \in U_{i} \cap U_{j} \cap \operatorname{Max} G$. An application of Lemma 3.5 yields $g \in G$ such that $[g]_{\mathfrak{p}}=\left[g_{i}\right]_{\mathfrak{p}}$ for each $i \in\{1, \ldots, n\}$ and each $\mathfrak{p} \in U_{i}$; in particular, $\widehat{g}(\mathfrak{m})=\widehat{g}_{i}(\mathfrak{m})$ for each $i \in\{1, \ldots, n\}$ and each $\mathfrak{m} \in Z_{i}$. To prove the uniqueness assertion, assume $\operatorname{Max} G=\bigcup_{i=1}^{n} Z_{i}$, ad suppose $g, g^{\prime} \in G$ satisfy $[g]_{\mathfrak{m}}=\left[g^{\prime}\right]_{\mathfrak{m}}=\left[g_{i}\right]_{\mathfrak{m}}$ for each $i \in\{1, \ldots, n\}$ and each $\mathfrak{m} \in Z_{i}$. Then $[g]_{\mathfrak{m}}=\left[g^{\prime}\right]_{\mathfrak{m}}$ for each $\mathfrak{m} \in \bigcup_{i=1}^{n} Z_{i}=\operatorname{Max} G$. Since $(G, u)$ is semisimple, we have $\operatorname{Rad} G=\{0\}$ by Lemma 2.4. Then the map $G \longrightarrow \prod_{\mathfrak{m} \in \operatorname{Max} G} G / \mathfrak{m}$ given by $g \longmapsto\left([g]_{\mathfrak{m}}\right)_{\mathfrak{m} \in \operatorname{Max} G}$ has trivial kernel, and thus is injective. It follows that $g=g^{\prime}$, as was to be shown. 
The strongly semisimple Chinese Remainder Theorem 3.6. Let $(G, u)$ be a unital $\ell$-group, and let $\left\{I_{i}\right\}_{i=1}^{n}$ be a finite collection of principal $\ell$-ideals of $G$. Suppose that $\left\{g_{i}\right\}_{i=1}^{n}$ is a finite subset of $G$ such that

$$
\left[g_{i}\right]_{\mathfrak{m}}=\left[g_{j}\right]_{\mathfrak{m}}
$$

for each $i, j \in\{1, \ldots, n\}$ and each maximal $\ell$-ideal $\mathfrak{m}$ that extends $I_{i} \vee I_{j}$. If $(G, u)$ is strongly semisimple, there exists an element $g \in G$ such that $[g]_{I_{i}}=\left[g_{i}\right]_{I_{i}}$ for each $i \in\{1, \ldots, n\}$.

Proof. This is a direct dualisation of the strongly semisimple Patching Lemma 3.5 to $G$, using Lemma 2.1 .

Example 3.7. Theorem 3.6 fails in the absence of strong semisimplicity, and hence so does the theorem stated in Section 1. Consider, for example, the lexicographic product $\mathbb{Z} \overrightarrow{\times} \mathbb{Z}$ of the additive group of integers with itself. Thus, $\mathbb{Z} \overrightarrow{\times} \mathbb{Z}$ is free Abelian of rank 2 as a group, and $(a, b) \geqslant\left(a^{\prime}, b^{\prime}\right)$ if either $a>a^{\prime}$, or else $a=a^{\prime}$ and then $b \geqslant b^{\prime}$. Now $\mathbb{Z} \overrightarrow{\times} \mathbb{Z}$ is not semisimple, and has exactly two $\ell$-ideals: the maximal $\ell$-ideal $I_{1}=:\{(0, b) \mid b \in \mathbb{Z}\}$, and the prime non-maximal $\ell$-ideal $I_{2}:=\{(0,0)\} \subseteq I_{1}$. They are evidently both principal. Consider the elements $g_{1}:=(0,0)$ and $g_{2}:=(0,1)$. Then $\left[g_{1}\right]_{I_{1}}=\left[g_{2}\right]_{I_{1}}$. However, there can be no $g \in \mathbb{Z} \overrightarrow{\times} \mathbb{Z}$ with $[g]_{I_{2}}=\left[g_{1}\right]_{I_{2}}=\left[g_{2}\right]_{I_{2}}$, simply because $\left[g_{1}\right]_{I_{2}}=g_{1} \neq g_{2}=\left[g_{2}\right]_{I_{2}}$.

\section{References}

[1] L. P. Belluce and A. Di Nola. Simplicial structures in MV-algebras and logic. J. Symbolic Logic, 72(2):584-600, 2007.

[2] A. Bigard, K. Keimel, and S. Wolfenstein. Groupes et anneaux réticulés. Lecture Notes in Mathematics, Vol. 608. Springer-Verlag, Berlin, 1977.

[3] R. L. O. Cignoli, I. M. L. D'Ottaviano, and D. Mundici. Algebraic foundations of many-valued reasoning, volume 7 of Trends in Logic-Studia Logica Library. Kluwer Academic Publishers, Dordrecht, 2000.

[4] P. M. Cohn. Universal algebra, volume 6 of Mathematics and its Applications. D. Reidel Publishing Co., Dordrecht, second edition, 1981.

[5] W. H. Cornish. The Chinese remainder theorem and sheaf representations. Fund. Math., 96(3):177-187, 1977.

[6] E. J. Dubuc and Y. A. Poveda. The Intimate Relationship Between the McNaughton and the Chinese Remainder Theorems for MV-algebras. Studia Logica, pages 1-3, 2012.

[7] E. J. Dubuc and Y. A. Poveda. Representation theory of MV-algebras. Ann. Pure Appl. Logic, 161(8):1024-1046, 2010.

[8] M. Erné, J. Koslowski, A. Melton, and G. E. Strecker. A primer on Galois connections. In Papers on general topology and applications (Madison, WI, 1991), volume 704 of Ann. New York Acad. Sci., pages 103-125. New York Acad. Sci., New York, 1993.

[9] A. R. Ferraioli and A. Lettieri. Representations of MV-algebras by sheaves. MLQ Math. Log. Q., 57(1):27-43, 2011.

[10] A. Filipoiu and G. Georgescu. Compact and Pierce representations of MV-algebras. Rev. Roumaine Math. Pures Appl., 40(7-8):599-618, 1995.

[11] M. Gehrke, S. van Gool, and V. Marra. Sheaf representations of MV-algebras and lattice-ordered abelian groups via duality. 2013. Manuscript. Available online at arXiv:1306.2839.

[12] L. Gillman and M. Jerison. Rings of continuous functions. The University Series in Higher Mathematics. D. Van Nostrand Co., Inc., Princeton, N.J.-Toronto-London-New York, 1960.

[13] A. W. Hager and L. C. Robertson. Representing and ringifying a Riesz space. In Symposia Mathematica, Vol. XXI (Convegno sulle Misure su Gruppi e su Spazi Vettoriali, Convegno sui Gruppi e Anelli Ordinati, INDAM, Rome, 1975), pages 411-431. Academic Press, London, 1977.

[14] M. Hochster. Prime ideal structure in commutative rings. Trans. Amer. Math. Soc., 142:43-60, 1969.

[15] O. Hölder. Die Axiome der Quantität und die Lehre vom Maß. Leipz. Ber., 53:1-64, 1901.

[16] P. T. Johnstone. Stone spaces, volume 3 of Cambridge Studies in Advanced Mathematics. Cambridge University Press, Cambridge, 1986. Reprint of the 1982 edition.

[17] K. Keimel. Représentation d'anneaux réticulés dans des faisceaux. C. R. Acad. Sci. Paris Sér. A-B, 266:A124-A127, 1968.

[18] P. H. Krauss and D. M. Clark. Global subdirect products. Mem. Amer. Math. Soc., 17(210):ii+109, 1979.

[19] D. Mundici. Interpretation of AF $C^{*}$ algebras in Lukasiewicz sentential calculus. Journal of Functional Analysis, 65:15-63, 1986.

[20] W. Rump and Y. C. Yang. Jaffard-Ohm correspondence and Hochster duality. Bull. Lond. Math. Soc., 40(2):263-273, 2008.

[21] N. Schwartz. Sheaves of abelian l-groups. Order. to appear. Published online 7 June 2012.

[22] D. J. Vaggione. Sheaf representation and Chinese Remainder Theorems. Algebra Universalis, 29:232-272, 1992.

[23] Y. Yang. l-Groups and Bézout Domains. Ph.D. Thesis. Universität Stuttgart, Holzgartenstr. 16, 70174, Stuttgart, 2006.

[24] K. Yosida. On vector lattice with a unit. Proc. Imp. Acad. Tokyo, 17:121-124, 1941.

[25] K. Yosida and M. Fukamiya. On vector lattice with a unit. II. Proc. Imp. Acad. Tokyo, 17:479-482, 1941. 